\title{
FLORÍSTICA EM UM HECTARE DE CERRADO STRICTO SENSU NA ARIE - CERRADO PÉ-DE-GIGANTE, SANTA RITA DO PASSA QUATRO, SP.
}

\author{
Veridiana de Lara Weiser ${ }^{1}$ \\ Silvana Aparecida Pires de Godoy ${ }^{2}$
}

Recebido em 21/03/00. Aceito em 16/04/01

\begin{abstract}
RESUMO - (Florística em um hectare de cerrado stricto sensu na ARIE - Cerrado Pé-de-Gigante, Santa Rita do Passa Quatro, SP). Foi realizado um levantamento florístico em um ha de cerrado stricto sensu, na parte norte da ARIE - Cerrado Pé-de-Gigante, Santa Rita do Passa Quatro, São Paulo. Foram coletados 428 espécimes em fase reprodutiva, em vinte e cinco excursões de coleta, durante o período de novembro de 1996 a abril de 1998. A listagem florística obtida apresenta 141 espécies, distribuídas em 109 gêneros e 49 famílias. As famílias mais representativas foram: Leguminosae, Asteraceae, Malpighiaceae e Myrtaceae. A floração e frutificação das espécies foram maiores no período chuvoso. Espécies zoocóricas (61) predominaram, seguidas pelas anemocóricas (46) e autocóricas (34). Analisou-se a flora como um todo e seus componentes herbáceo-subarbustivo e arbustivo-arbóreo, separadamente.
\end{abstract}

Palavras chaves: cerrado, levantamento florístico, fenologia, síndromes de dispersão.

\begin{abstract}
Floristic composition in a hectare of cerrado stricto sensu hectare in the ARIE - Cerrado Péde-Gigante, Santa Rita do Passa Quatro, SP). A floristic survey was carried out in one ha of cerrado stricto sensu, in the northern part of the ARIE - Cerrado Pé-de-Gigante, Santa Rita do Passa Quatro, São Paulo, Brazil. A total of 428 specimens in reproductive stage were collected in twenty-five field trips to the area, from november 1996 to april 1998. The flora shows 141 species, representing 109 genera and 49 families. Leguminosae, Asteraceae, Malpighiaceae and Myrtaceae were the most representative families. Flowering and fruiting were higher in the rainy season. The zoochorous species (61) were greater than anemo (46) and autochorous (34) ones. The cerrado flora as a whole and its two components, woody and herbaceous, were analysed separately.
\end{abstract}

Key words - cerrado, floristic inventory, phenology and dispersal syndromes.

\section{Introdução}

O Estado de São Paulo ainda apresenta áreas de vegetação natural, representadas pelas matas ciliares e de galeria, cerrado, mata atlântica, restingas e mangues, o que torna relevante o estudo dessas áreas, como con- tribuição ao conhecimento da flora e seu melhor aproveitamento.

O levantamento florístico é um dos estudos iniciais para o conhecimento da flora de uma determinada área e implica na produção de

\footnotetext{
${ }^{1}$ Bolsista de Iniciação Científica FAPESP - Proc. 97/07302-8.

${ }^{2}$ Departamento de Biologia - Faculdade de Filosofia, Ciências e Letras de Ribeirão Preto - USP. Av. Bandeirantes 3900 CEP 14040-030 - Ribeirão Preto, SP.
} 
uma lista das espécies ali instaladas, sendo de fundamental importância a correta identificação taxonômica dos espécimes e a manutenção de exsicatas em herbário, que poderão contribuir para o estudo dos demais atributos da comunidade (Martins, 1990).

No estado de São Paulo, uma das formações que tem merecido a atenção dos pesquisadores quanto ao levantamento florístico é o cerrado, dominante no Brasil Central e que ocorre como manchas em partes do Estado. O cerrado ocupa dois milhões de quilômetros quadrados, o que representa cerca de $23 \%$ do território brasileiro (Ratter et al., 1997), sendo considerado o segundo maior bioma do país em área (Ribeiro \& Walter, 1998).

Alguns levantamentos realizados no Estado de São Paulo foram os de Eiten (1963), em Mogi Guaçu; Oliveira e Souza (1977), em Itirapina; Silberbauer-Gottsberger et al. (1977), em Botucatu; Toledo Filho et al. (1984), em Moji Mirim; Pagano et al. (1989), em Corumbataí; Mantovani \& Martins (1993), em Moji Guaçu; Batalha et al. (1997a), em Pirassununga e Batalha (1997), em Santa Rita do Passa Quatro.

Em 1999, Castro et al. compilaram diversos levantamentos florísticos e fitossociológicos, relacionando 1709 espécies para a flora do cerrado.

Castro (1987) iniciou o levantamento florístico e fitossociológico da mancha de cerrado em questão. A área estudada pelo autor correspondia à parte sudeste da antiga Gleba Pé-de-Gigante e caracterizava-se por portar um cerrado denso, rico em arvoretas e com maior freqüência de plantas com caules suberosos que rugosos e lisos.

Batalha (1997) realizou o levantamento florístico na vegetação da ARIE - Cerrado Péde-Gigante, no intuito de distinguir as diferentes formações e fisionomias, além de contribuir com estudos fitogeográficos sobre o cerrado.
O levantamento florístico em área demarcada abre perspectivas para o desenvolvimento de pesquisas relacionadas à fitossociologia, à fenologia e à dinâmica das populações ali instaladas. Do mesmo modo, o amplo conhecimento da flora do Cerrado é um importante subsídio no planejamento e implementação de áreas representativas do bioma, que devem ser priorizadas para conservação e manejo racional (Felfili et al., 1993 e Mendonça et al., 1998).

Este trabalho teve como objetivo o levantamento florístico em um hectare de cerrado stricto sensu, na parte norte da Área de Relevante Interesse Ecológico (ARIE) - Cerrado Pé-de-Gigante, localizada no município de Santa Rita do Passa Quatro, São Paulo.

\section{Material e métodos}

A Área de Relevante Interesse Ecológico (ARIE) - Cerrado Pé-de-Gigante foi criada no local da antiga Gleba Pé-de-Gigante, parte do Parque Estadual de Vassununga até 1971, pelo Decreto Federal $n^{0} 99.275$ de 06.06.90. Localiza-se no município de Santa Rita do Passa Quatro, Estado de São Paulo, à margem esquerda da Rodovia Anhangüera, entre os quilômetros 254 e 259, em área de cerca de 1060,03 ha, coordenadas geográficas $21^{\circ} 43^{\prime} \mathrm{S}$ e $47^{\circ} 35^{\prime} \mathrm{W}$, altitude de $600 \mathrm{~m}$, sob o clima Cwag' de Köppen (Batalha, 1997) e sobre latossolo vermelho-amarelo fase arenosa (Castro, 1987).

Segundo Batalha (1997), a ARIE apresenta as seguintes fisionomias de cerrado: cerradão, cerrado stricto sensu (s.s.), campo cerrado, campo sujo, campo úmido e ainda as formações: mata ciliar e floresta estacional semidecídua.

Durante o período de novembro de 1996 a abril de 1998, foram feitas coletas sistemáticas de material em estágio reprodutivo, na área de estudo proposta, que corresponde à parte norte da ARIE - Cerrado Pé-de-Gigante 
e tem fisionomia de cerrado s.s. Foram anotadas as observações sobre as formas de vida, síndromes de dispersão e os períodos de floração e frutificação das espécies.

Para classificar as formas de vida e as síndromes de dispersão no campo, adotouse a chave proposta por Mantovani (1983) e o sistema de classificação de Pijl (1982), respectivamente. Quando não foi possível observar e coletar os frutos dos espécimes, as síndromes de dispersão foram classificadas de acordo com as descritas na literatura por: Mantovani \& Martins (1993) e Batalha et al. (1997a).

O sistema de classificação adotado foi o de Cronquist $(1981,1988)$, exceto para as famílias Caesalpiniaceae, Fabaceae e Mimosaceae, que foram tratadas como subfamílias da família Leguminosae. As espécies foram identificadas através de bibliografia pertinente e confirmadas por especialistas ou por comparação com as coleções do herbário SPF da Universidade de São Paulo. Os espécimes floridos foram incorporados à coleção ARIE - Cerrado Pé-de-Gigante, e os frutos à carpoteca do herbário do Campus da Faculdade de Filosofia, Ciências e Letras de Ribeirão Preto (SPFR).

\section{Resultados e discussão}

Foram coletados 428 espécimes em fase reprodutiva, representando 141 espécies, distribuídas em 109 gêneros e 49 famílias. A Tabela 1 apresenta a flora obtida, obedecendo à ordem alfabética de famílias, gêneros e espécies, dentro do grupo de Magnoliophyta e suas subdivisões, Magnoliopsida e Liliopsida.

Analisando a flora como um todo, das 49 famílias amostradas, $25(51 \%)$ apresentaram uma única espécie, oito $(16 \%)$ apresentaram duas espécies e cinco (10\%) apresentaram três espécies (Fig.1). No entanto, as onze restantes: Leguminosae, Asteraceae, Malpighiaceae,
Myrtaceae, Rubiaceae, Annonaceae, Apocynaceae, Bignoniaceae, Vochysiaceae, Melastomataceae e Sapindaceae, compreendem cerca de $60 \%$ do total das espécies (Fig.2), o que significa que menos de um terço do número de famílias concentra os maiores valores da riqueza de espécies. A representatividade dessas famílias também foi verificada por: Warming (1892), em Lagoa Santa; Goodland (1969), no Triângulo Mineiro; Mantovani e Martins (1993), em Moji Guaçu; Batalha et al.(1997a), em Pirassununga e Batalha (1997), em Santa Rita do Passa Quatro.

Dos 109 gêneros amostrados, 91 (83\%) apresentaram uma única espécie, sete $(6 \%)$ apresentaram duas espécies e oito (7\%) apresentaram três espécies. Byrsonima, Eugenia e Qualea foram os gêneros mais representados, com quatro $(3 \%)$ espécies cada.

O componente arbustivo-arbóreo compreende as fanerófitas e caméfitas ou fanerófitas, enquanto que o componente herbáceosubarbustivo, as caméfitas, epífitas, geófitas, hemicriptófitas, lianas, terófitas, parasitas e semiparasitas vasculares. As duas últimas formas de vida não foram representadas neste estudo.

Analisando o componente arbustivo-arbóreo, constituído por 70 espécies, 18 (64\%) famílias apresentaram uma única espécie (Fig. 1). Leguminosae, Myrtaceae, Malpighiaceae, Vochysiaceae, Annonaceae e Melastomataceae foram as famílias com maiores valores de riqueza nesse componente, totalizando $61 \%$ das espécies (Fig. 3). A representatividade dessas famílias nesse componente também foi verificada na Reserva Biológica de Moji Guaçu (Mantovani \& Martins, 1993) e no cerrado lato sensu da ARIE - Cerrado Pé-de-Gigante (Batalha, 1997). Ressalta-se, ainda, que do total de famílias amostradas na flora como um todo, 17 (35\%) foram exclusivas desse componente. Analisando o componente herbáceo- 


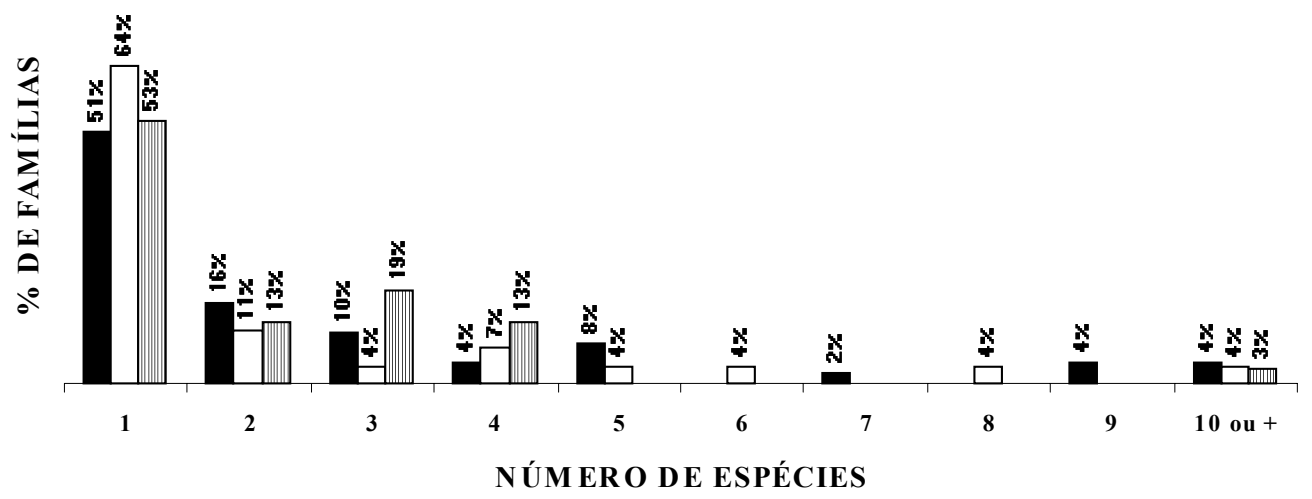

G FLORA COMO UM TODO

$\square$ COMPONENTE ARB US TIVO-ARBÓREO

四COMPONENTE HERB ÁCEO-S UB ARB US TIVO

Figura 1 - Distribuição, em porcentagem, das famílias pelo número de espécies da flora como um todo e dos componentes arbustivo-arbóreo e herbáceo-subarbustivo, em um ha de cerrado s.s. da ARIE - Cerrado Pé-de-Gigante, Santa Rita do Passa Quatro, SP.

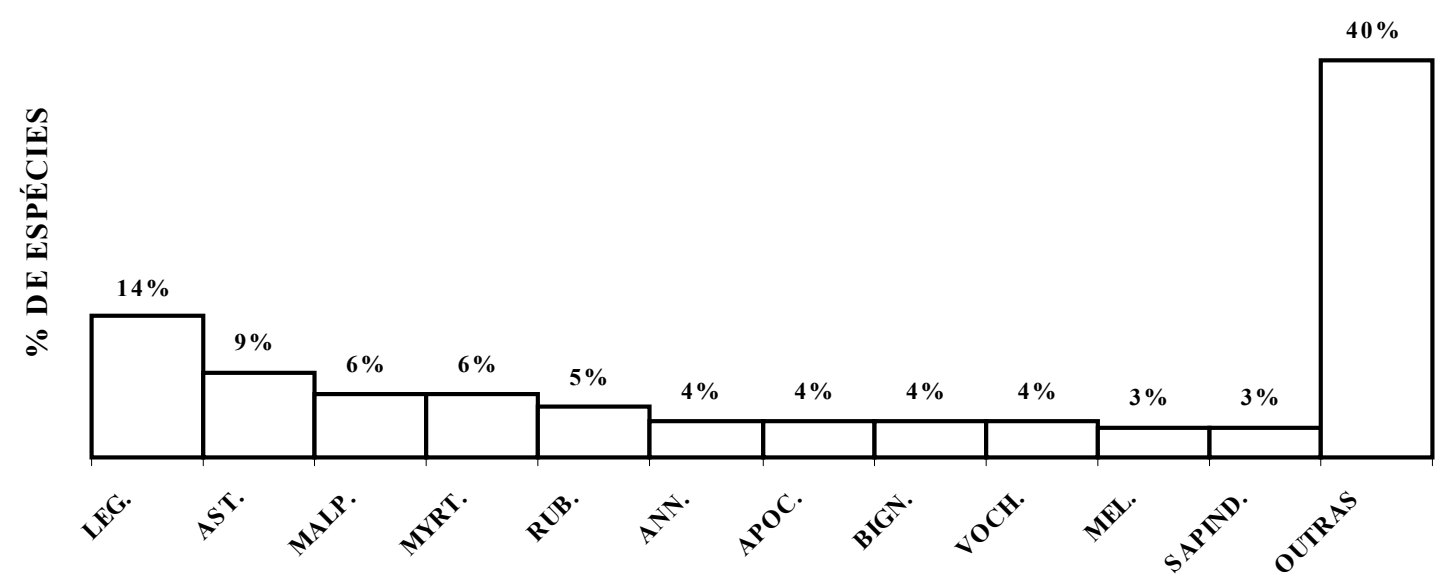

Figura 2 - Distribuição, em porcentagem, das espécies nas famílias mais representadas, em um ha de cerrado s.s. da ARIE Cerrado Pé-de-Gigante, Santa Rita do Passa Quatro, SP.

subarbustivo, constituído por 71 espécies, 17 (53\%) famílias apresentaram uma única espécie (Fig. 1). Asteraceae, Bignoniaceae, Leguminosae, Rubiaceae e Sapindaceae foram as famílias com maiores valores de riqueza nesse componente, totalizando $39 \%$ das espécies (Fig. 4). A representatividade dessas famílias nesse componente também foi verificada no cerrado lato sensu da ARIE Cerrado Pé-de-Gigante (Batalha, 1997) e com exceção de Sapindaceae, na Reserva Biológica de Moji Guaçu (Mantovani \& Martins, 1993). É importante salientar que do total de famílias amostradas na flora como um todo, $21(42 \%)$ foram exclusivas desse componente.

Comparando-se os números de espécies obtidas nos componentes herbáceosubarbustivo e arbustivo-arbóreo da área de estudo, observou-se a relação de 1:1. 


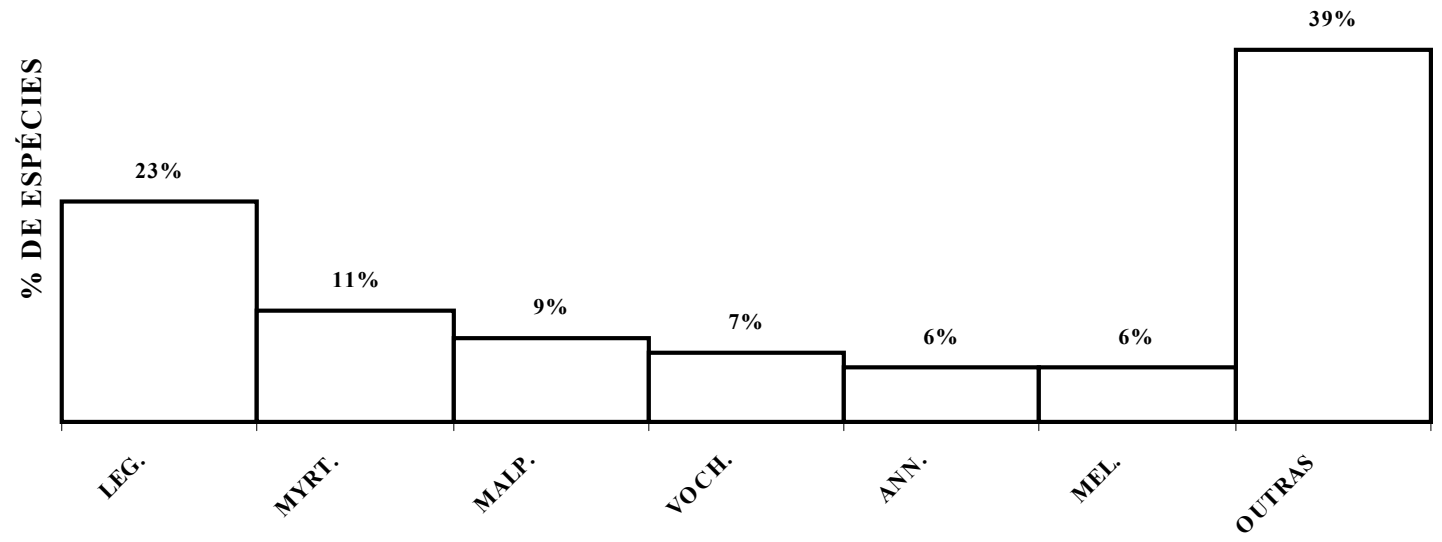

Figura 3 - Distribuição, em porcentagem, das espécies do componente arbustivo-arbóreo nas famílias mais representadas, em um ha de cerrado s.s. da ARIE - Cerrado Pé-de-Gigante, Santa Rita do Passa Quatro, SP.

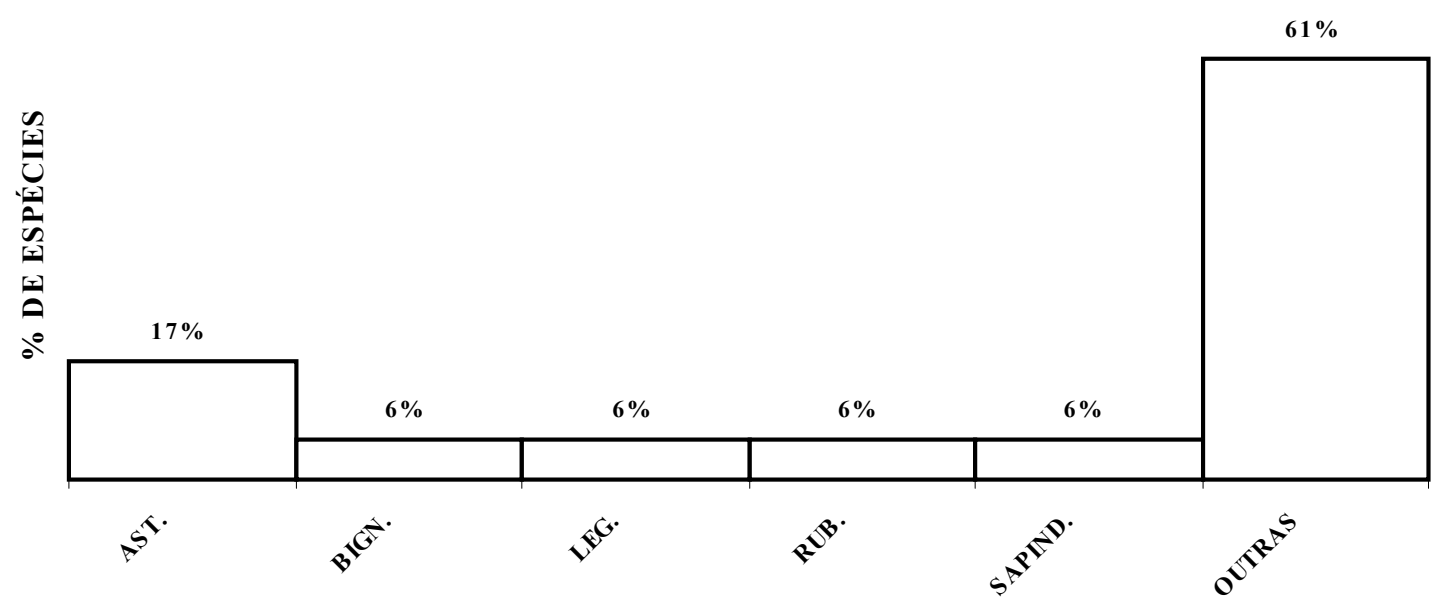

Figura 4 - Distribuição, em porcentagem, das espécies do componente herbáceo-subarbustivo nas famílias mais representadas, em um ha de cerrado s.s. da ARIE - Cerrado Pé-de-Gigante, Santa Rita do Passa Quatro, SP.

Analisando a flora como um todo, 30 (21\%) espécies, a maioria pertencente ao componente arbustivo-arbóreo, foram encontradas apenas em estágio vegetativo durante o período de coleta. Além disso, muitas espécies herbáceo-subarbustivas não foram coletadas na fenofase de fruto pois, conforme observado por Batalha (1997), apresentam um ciclo epígeo de poucas semanas.

O número de espécies observadas em floração e/ou frutificação da flora como um todo e de seus componentes arbustivo-arbóreo e herbáceo-subarbustivo, foi maior no período chuvoso, que segundo Batalha (1997), compreende os meses de setembro a maio. $\mathrm{Na}$ flora como um todo, a floração atingiu seu valor máximo nos meses de setembro e novembro e a frutificação, nos meses de novembro e fevereiro (Fig. 5). No componente arbustivo-arbóreo, a floração atingiu seu valor máximo nos meses de setembro a novembro e a frutificação, nos meses de novembro e fevereiro (Fig. 5), enquanto que no componente herbáceo-subarbustivo, a floração foi mais elevada no mês de janeiro e a frutificação no mês de junho (Fig. 5). 

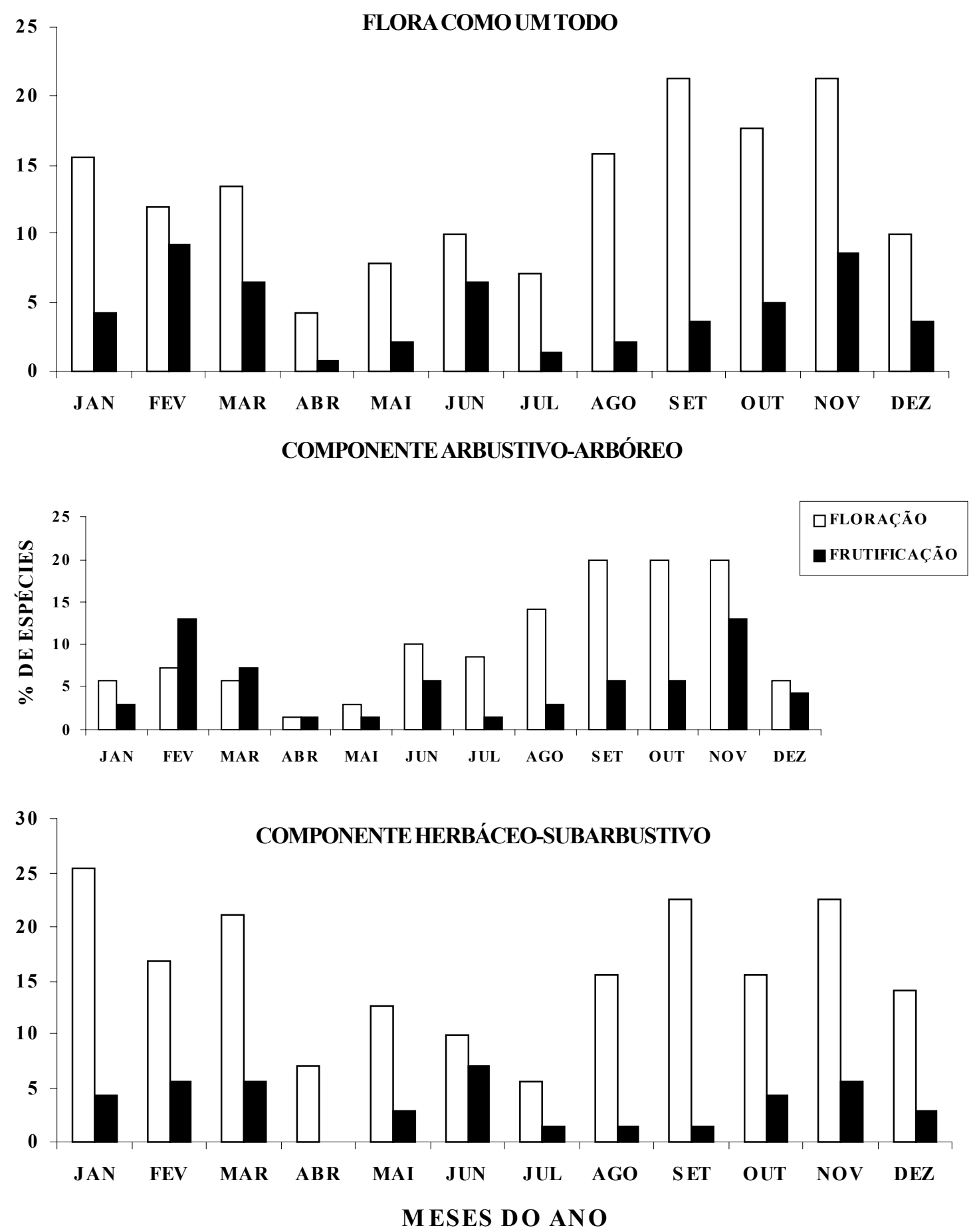

Figura 5 - Distribuição, em porcentagem, das espécies da flora como um todo e dos componentes arbustivo-arbóreo e herbáceo subarbustivo em floração e frutificação, ao longo do ano, em um ha de cerrado s.s. da ARIE - Cerrado Pé-de-Gigante, Santa Rita do Passa Quatro, SP. 


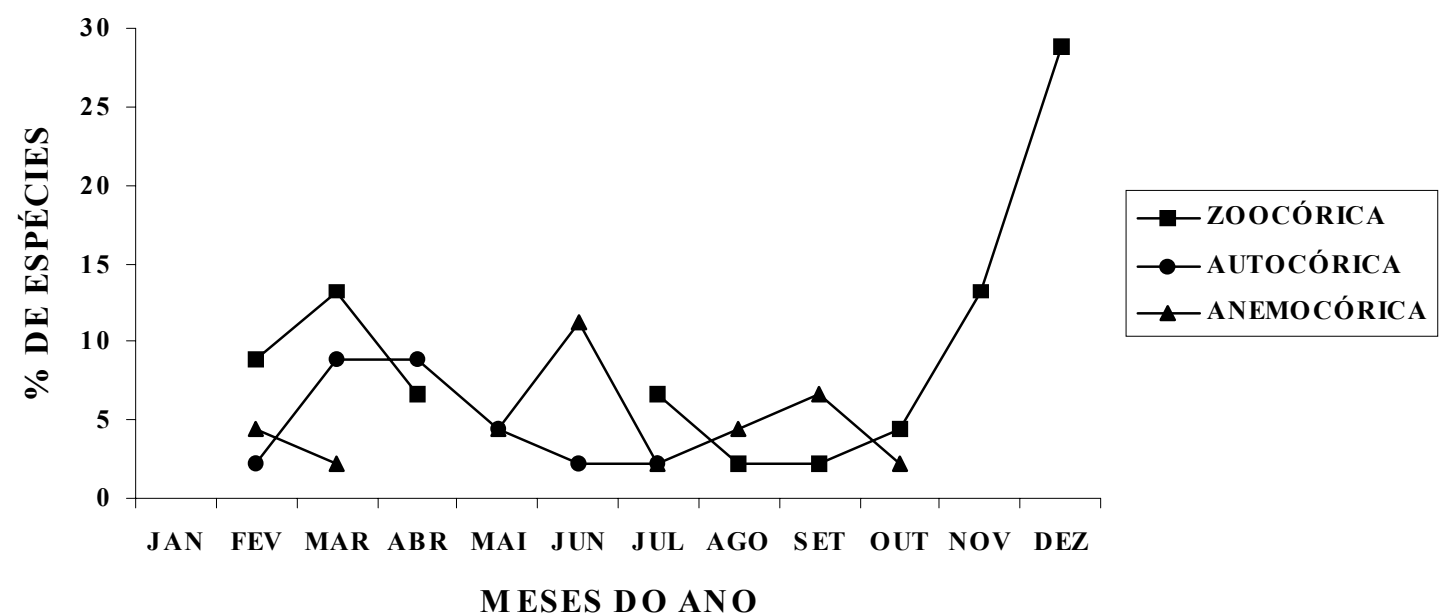

Figura 6 - Distribuição, em porcentagem, das espécies que frutificaram ao longo do ano, segundo a forma de dispersão, em um ha de cerrado s.s. da ARIE - Cerrado Pé-de-Gigante, Santa Rita do Passa Quatro, SP.

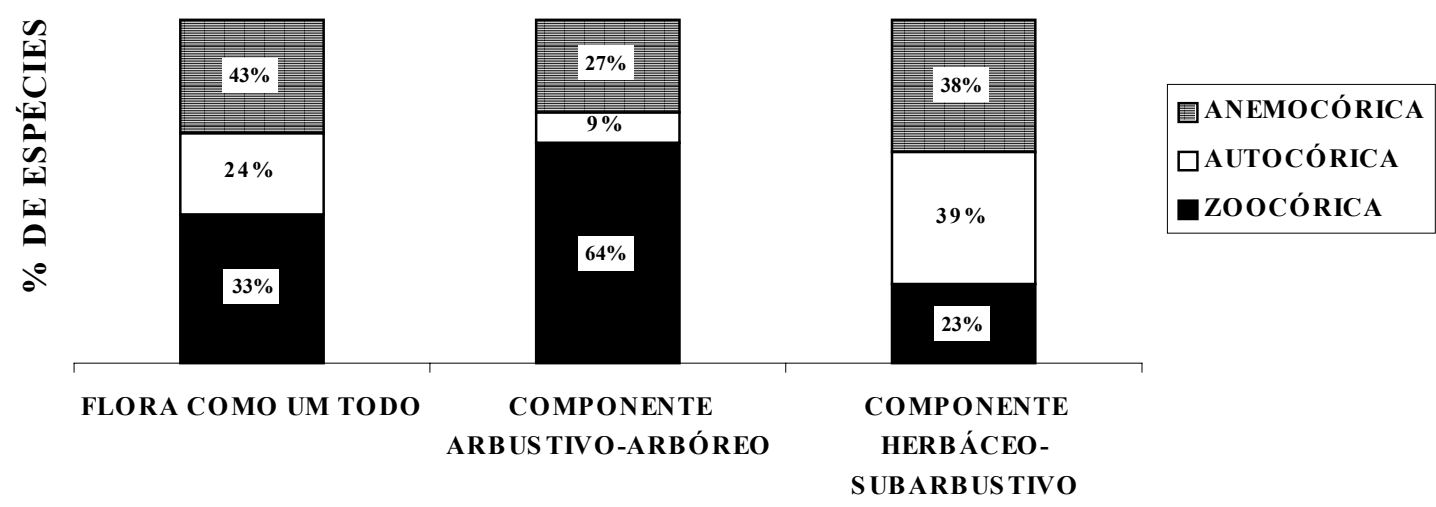

Figura 7 - Distribuição, em porcentagem, das síndromes de dispersão das espécies na flora como um todo e nos componentes arbustivo-arbóreo e herbáceo-subarbustivo, em um ha de cerrado s.s. da ARIE - Cerrado Pé-de-Gigante, Santa Rita do Passa Quatro, SP.

A análise da fenologia de frutificação das espécies com diferentes síndromes de dispersão mostrou a predominância da frutificação de espécies zoocóricas e autocóricas no início e no final da estação chuvosa, respectivamente e a de espécies anemocóricas, na estação seca (Fig.6). Este padrão para espécies zoocóricas e anemocóricas foi verificado por Mantovani \& Martins (1988), em Mogi Guaçu; Batalha et al. (1997b), em Pirassununga; Oliveira, (1998) e Batalha \& Mantovani (2000), no cerrado lato sensu da ARIE - Cerrado Pé-de-Gigante.
Do total de espécies amostradas, 61 (43\%) foram classificadas como zoocóricas, 46 (32\%), anemocóricas e 34 (24\%), autocóricas. No componente arbustivo-arbóreo predominaram as espécies zoocóricas (64\%) e no componente herbáceo-subarbustivo, as espécies autocóricas (39\%) e anemocóricas (38\%) (Fig. 7). Este padrão foi encontrado por Batalha et al. (1997b), em Pirassununga e Batalha (1997), no cerrado lato sensu da ARIE Cerrado Pé-de-Gigante. 
Tabela 1 - Lista de espécies amostradas em 1 ha de cerrado s.s. da ARIE - Cerrado Pé-de-Gigante, Santa Rita do Passa Quatro, SP. Legenda: $(*)=$ espécies observadas no campo em estágio vegetativo; forma de vida - CAM = caméfita, CAM-FAN = caméfita ou fanerófita, EPI = epífita, FAN = fanerófita, GEO = geófita, HEM = hemicriptófita, LIA = liana e TER = terófita; síndromes de dispersão - ANE = anemocórica, AUT = autocórica e ZOO = zoocórica; período de floração e frutificação - JAN $=$ janeiro, $\mathrm{FEV}=$ fevereiro,..., $\mathrm{NOV}=$ novembro e DEZ $=$ dezembro. $\mathrm{O}$ hífen (-) representa continuidade entre os meses.

\begin{tabular}{|c|c|c|c|c|c|}
\hline FAMÍLIA / ESPÉCIE & $\begin{array}{c}\text { Forma } \\
\text { de } \\
\text { vida }\end{array}$ & $\begin{array}{c}\text { Síndrome } \\
\text { de } \\
\text { dispersão }\end{array}$ & $\begin{array}{c}\text { Período } \\
\text { de } \\
\text { floraçãao }\end{array}$ & $\begin{array}{c}\text { Período } \\
\text { de } \\
\text { frutificação }\end{array}$ & $\begin{array}{l}\text { Número do } \\
\text { coletor } \\
\text { SAPGodoy }\end{array}$ \\
\hline \multicolumn{6}{|l|}{$\begin{array}{l}\text { MAGNOLIOPHYTA } \\
\text { MAGNOLIOPSIDA }\end{array}$} \\
\hline \multicolumn{6}{|l|}{ Amaranthaceae } \\
\hline Alternanthera brasiliana (L.) Kuntze & HEM & AUT & JAN & - & 801 \\
\hline Froelichia lanata Moq. & HEM & AUT & NOV-JAN & - & 1179 \\
\hline \multicolumn{5}{|l|}{ Anacardiaceae } & 1338 \\
\hline Anacardium humile A.St.-Hil. & CAM & $\mathrm{ZOO}$ & AGO-SET & - & 1048 \\
\hline $\begin{array}{l}\text { Tapirira guianensis Aubl. } \\
\text { Annonaceae }\end{array}$ & CAM & $\mathrm{ZOO}$ & MAI & - & 827 \\
\hline Annona crassiflora Mart. & FAN & $\mathrm{ZOO}$ & NOV & - & 1171 \\
\hline Annona dioica A.St.-Hil. & CAM & $\mathrm{ZOO}$ & NOV & - & 1213 \\
\hline Annona sp & FAN & $\mathrm{ZOO}$ & - & FEV & 1320 \\
\hline Duguetia furfuracea (A.St.-Hil.) Benth. \& Hook.f. & FAN & $\mathrm{ZOO}$ & OUT-JAN & OUT-MAR & 1284 \\
\hline $\begin{array}{l}\text { Xylopia aromatica }(\text { Lam.) Mart. } \\
\text { Apocynaceae }\end{array}$ & \multicolumn{4}{|c|}{ Apocynaceae } & 849 \\
\hline Aspidosperma tomentosum Mart. (*) & FAN & ANE & - & - & - \\
\hline Forsteronia glabrescens Müll.Arg. & LIA & ANE & OUT & MAI-JUN & 1142 \\
\hline Hancornia speciosa Gomes $(*)$ & FAN & $\mathrm{ZOO}$ & - & - & - \\
\hline Odontadenia lutea (Vell.) Markgr. (*) & LIA & ANE & - & - & - \\
\hline \multicolumn{6}{|l|}{ Araliaceae } \\
\hline \multicolumn{3}{|l|}{ Asclepiadaceae } & JUN & JUN-SET & 837 \\
\hline Astephanus carassensis Malme & LIA & ANE & FEV-MAR & - & 1339 \\
\hline \multicolumn{6}{|l|}{ Asteraceae } \\
\hline Eremanthus sphaerocephalus (DC.) Backer & HEM & ANE & SET & - & 1262 \\
\hline Eupatorium squalidum DC. & TER & ANE & MAR-MAI & - & 1334 \\
\hline Eupatorium $\mathrm{sp}$ & HEM & ANE & NOV & - & 1216 \\
\hline Gochnatia barrosoi Cabrera $(*)$ & CAM & ANE & - & - & - \\
\hline Gochnatia pulchra Cabrera & CAM & ANE & - & JUN & 846 \\
\hline Kanimia oblongifolia (DC.) Backer & HEM & ANE & NOV & - & 1158 \\
\hline Senecio sp & TER & ANE & AGO & - & 938 \\
\hline Trichogonia salviifolia Gardner & HEM & ANE & FEV & - & 1290 \\
\hline Vernonia bardanoides Less. & HEM & ANE & MAR & - & 1342 \\
\hline Vernonia onopordioides Baker & HEM & ANE & MAR & - & 1355 \\
\hline Vernonia rubriramea Mart. & CAM & ANE & JUN & - & 898 \\
\hline Viguiera arenaria Baker & HEM & AUT & JAN & - & 793 \\
\hline \multicolumn{6}{|l|}{ Bignoniaceae } \\
\hline Distictella elongata (Vahl) Urb. & LIA & ANE & NOV-DEZ & FEV & 1218 \\
\hline Jacaranda decurrens Cham. & CAM & ANE & AGO-SET & - & 985 \\
\hline Memora peregrina (Miers) Sandwith & CAM & ANE & SET-JAN & - & 1015 \\
\hline Pyrostegia venusta (Ker Gawl.) Miers & LIA & ANE & JUN-AGO & - & 861 \\
\hline \multicolumn{6}{|l|}{ Bombacaceae } \\
\hline $\begin{array}{c}\text { Eriotheca gracilipes (K.Schum.) A.Robyns } \\
\text { Burseraceae }\end{array}$ & FAN & ANE & JUN-AGO & AGO-SET & 857 \\
\hline $\begin{array}{c}\text { Protium heptaphyllum (Aubl.) Marchal }(*) \\
\text { Caryocaraceae }\end{array}$ & FAN & $\mathrm{ZOO}$ & - & - & - \\
\hline Caryocar brasiliense Cambess. & FAN & $\mathrm{ZOO}$ & OUT & NOV & 1107 \\
\hline
\end{tabular}




\begin{tabular}{|c|c|c|c|c|c|}
\hline FAMÍLIA / ESPÉCIE & $\begin{array}{l}\text { Forma } \\
\text { de } \\
\text { vida }\end{array}$ & $\begin{array}{l}\text { Síndrome } \\
\text { de } \\
\text { dispersão }\end{array}$ & $\begin{array}{l}\text { Período } \\
\text { de } \\
\text { floração }\end{array}$ & $\begin{array}{l}\text { Período } \\
\text { de } \\
\text { frutificação }\end{array}$ & $\begin{array}{l}\text { Número do } \\
\text { coletor } \\
\text { SAPGodoy }\end{array}$ \\
\hline $\begin{array}{c}\text { Celastraceae } \\
\text { Austroplenckia populnea (Reissek) Lundell }(*) \\
\text { Chrysobalanaceae }\end{array}$ & FAN & ANE & - & - & - \\
\hline Couepia grandiflora (Mart. \& Zucc.) Benth. ex Hook.f. & FAN & $\mathrm{ZOO}$ & SET-OUT & NOV-FEV & 1287 \\
\hline $\begin{array}{l}\text { Licania humilis Cham \& Schltdl. } \\
\text { Connaraceae }\end{array}$ & CAM & $\mathrm{ZOO}$ & AGO-SET & - & 972 \\
\hline Connarus suberosus Planch. $(*)$ & FAN & $\mathrm{ZOO}$ & - & - & - \\
\hline $\begin{array}{l}\text { Rourea induta Planch. } \\
\text { Cucurbitaceae }\end{array}$ & CAM & $\mathrm{ZOO}$ & SET & OUT-JAN & 994 \\
\hline $\begin{array}{c}\text { Cayaponia espelina (Silva Manso) Cogn. } \\
\text { Dilleniaceae }\end{array}$ & LIA & $\mathrm{ZOO}$ & NOV & - & 1224 \\
\hline $\begin{array}{c}\text { Davilla rugosa Poir. }(*) \\
\text { Ebenaceae }\end{array}$ & LIA & AUT & - & - & - \\
\hline $\begin{array}{r}\text { Diospyros hispida A.DC. }(*) \\
\text { Erythroxylaceae }\end{array}$ & FAN & $\mathrm{ZOO}$ & - & - & - \\
\hline $\begin{array}{c}\text { Erythroxylum suberosum A.St.-Hil. }(*) \\
\text { Euphorbiaceae }\end{array}$ & FAN & $\mathrm{ZOO}$ & - & - & - \\
\hline Croton sclerocalyx Müll.Arg. & HEM & AUT & JAN & - & 805 \\
\hline Manihot caerulescens Pohl & HEM & AUT & NOV & - & 1217 \\
\hline $\begin{array}{r}\text { Sapium glandulatum (Vell.) Pax } \\
\text { Hippocrateaceae }\end{array}$ & HEM & AUT & - & FEV & 1291 \\
\hline $\begin{array}{c}\text { Tontelea micrantha (Mart.) A.C.Sm. } \\
\text { Lamiaceae }\end{array}$ & FAN & $\mathrm{ZOO}$ & SET & - & 1076 \\
\hline Hyptis mutabilis Briq. & HEM & AUT & AGO-SET & - & 946 \\
\hline Hyptis reticulata Mart. & HEM & AUT & JAN-OUT & - & 825 \\
\hline $\begin{array}{r}\text { Peltodon tomentosus Pohl } \\
\text { Lauraceae }\end{array}$ & HEM & AUT & MAR & - & 1361 \\
\hline Ocotea corymbosa (Meisn.) Mez & FAN & $\mathrm{ZOO}$ & - & SET & 1045 \\
\hline $\begin{array}{l}\text { Ocotea pulchella Mart. } \\
\text { Leguminosae - Caesalpinioideae }\end{array}$ & FAN & $\mathrm{ZOO}$ & OUT-NOV & JUN & 848 \\
\hline Bauhinia rufa (Bong.) Steud. & FAN & AUT & OUT-FEV & - & 1134 \\
\hline Chamaecrista debilis (Vogel) H.S.Irwin \& Barneby & CAM & AUT & DEZ-JAN & - & 789 \\
\hline Chamaecrista desvauxii (Collad.) Killip & CAM & AUT & JAN-MAR & FEV & 1319 \\
\hline Chamaecrista serpens (L.) Greene & HEM & AUT & DEZ-JAN & - & 1228 \\
\hline Copaifera langsdorfii Desf. & FAN & $\mathrm{ZOO}$ & - & MAR & 1334 \\
\hline Dimorphandra mollis Benth. $(*)$ & FAN & $\mathrm{ZOO}$ & - & - & - \\
\hline Diptychandra aurantiaca Tul. & FAN & ANE & OUT-NOV & FEV & 1143 \\
\hline Hymenaea stigonocarpa Mart. ex Hayne $\left(^{*}\right)$ & FAN & $\mathrm{ZOO}$ & - & - & - \\
\hline Sclerobium paniculatum Vogel $(*)$ & FAN & ANE & - & - & - \\
\hline $\begin{array}{r}\text { Senna rugosa (G.Don) H.S.Irwin \& Barneby } \\
\text { Leguminosae - Faboideae }\end{array}$ & FAN & AUT & MAR & - & 1332 \\
\hline Crotalaria vitelina Ker Gawl. & HEM & AUT & JAN & - & 1283 \\
\hline Dalbergia miscolobium Benth. (*) & FAN & ANE & - & - & - \\
\hline Machaerium acutifolium Vogel & FAN & ANE & - & FEV & 1312 \\
\hline Pterodon emarginatus Vogel $(*)$ & FAN & ANE & - & - & - \\
\hline $\begin{array}{r}\text { Vatairea macrocarpa }(\text { Benth.) Ducke }(*) \\
\text { Leguminosae - Mimosoideae }\end{array}$ & FAN & ANE & - & - & - \\
\hline Anadenanthera falcata (Benth.) Speg. & FAN & AUT & SET & FEV-JUN & 852 \\
\hline Plathymenia reticulata Benth.(*) & FAN & ANE & - & - & - \\
\hline Stryphnodendron adstringens (Mart.) Coville $(*)$ & FAN & $\mathrm{ZOO}$ & - & - & - \\
\hline Stryphnodendron obovatum Benth. & FAN & AUT & - & MAR & 1357 \\
\hline $\begin{array}{c}\text { Stryphnodendron polyphyllum Mart. }(*) \\
\text { Lythraceae }\end{array}$ & FAN & AUT & - & 一 & - \\
\hline $\begin{array}{c}\text { Cuphea carthagenensis (Jacq.) J.F.Macbr. } \\
\text { Malpighiaceae }\end{array}$ & CAM & AUT & NOV-JAN & - & 1225 \\
\hline Banisteriopsis anisandra (A.Juss.) B.Gates \& & & & & & \\
\hline W.R.Anderson & LIA & ANE & AGO & - & 945 \\
\hline Banisteriopsis pubipetala (A.Juss.) Cuatrec. & LIA & ANE & JUN-SET & MAI-OUT & 907 \\
\hline Banisteriopsis stellaris (Griseb.) B.Gates & LIA & ANE & JAN-JUN & - & 908 \\
\hline Byrsonima coccolobifolia Kunth $(*)$ & FAN & $\mathrm{ZOO}$ & - & - & - \\
\hline Byrsonima crassa Nied. & FAN & $\mathrm{ZOO}$ & JAN-FEV & - & 1259 \\
\hline Byrsonima intermedia A.Juss. & FAN & $\mathrm{ZOO}$ & SET-MAR & NOV & 1023 \\
\hline
\end{tabular}




\begin{tabular}{|c|c|c|c|c|c|}
\hline FAMÍLIA / ESPÉCIE & $\begin{array}{c}\text { Forma } \\
\text { de } \\
\text { vida }\end{array}$ & $\begin{array}{c}\text { Síndrome } \\
\text { de } \\
\text { dispersão }\end{array}$ & $\begin{array}{c}\text { Período } \\
\text { de } \\
\text { floração }\end{array}$ & $\begin{array}{c}\text { Período } \\
\text { de } \\
\text { frutificação }\end{array}$ & $\begin{array}{l}\text { Número do } \\
\text { coletor } \\
\text { SAPGodoy }\end{array}$ \\
\hline Byrsonima verbascifolia (L.) Rich. ex A.Juss. & FAN & $\mathrm{ZOO}$ & MAR & - & 1356 \\
\hline Heteropterys byrsonimifolia A.Juss. & FAN & ANE & AGO-SET & SET & 937 \\
\hline $\begin{array}{l}\text { Heteropterys umbellata A.Juss. } \\
\text { Melastomataceae }\end{array}$ & FAN & ANE & OUT & - & 1151 \\
\hline Leandra lacunosa Cogn. & FAN & ANE & JUN-NOV & - & 909 \\
\hline Miconia albicans (Sw.) Triana & CAM-FAN & $\mathrm{ZOO}$ & JUN-SET & OUT-DEZ & 896 \\
\hline Miconia ligustroides (DC.) Naudin $(*)$ & FAN & $\mathrm{ZOO}$ & - & - & - \\
\hline $\begin{array}{l}\text { Miconia stenostachya DC. } \\
\text { Menispermaceae }\end{array}$ & CAM-FAN & $\mathrm{ZOO}$ & - & NOV & 1210 \\
\hline $\begin{array}{l}\text { Cissampelos ovalifolia DC. } \\
\text { Moraceae }\end{array}$ & HEM & AUT & NOV & - & 1184 \\
\hline $\begin{array}{l}\text { Ficus citrifolia Mill. } \\
\text { Myristicaceae }\end{array}$ & FAN & $\mathrm{ZOO}$ & NOV & FEV & 1207 \\
\hline $\begin{array}{r}\text { Virola sebifera Aubl. }(*) \\
\text { Myrtaceae }\end{array}$ & FAN & $\mathrm{ZOO}$ & - & - & - \\
\hline Campomanesia pubescens (DC.) O.Berg & FAN & $\mathrm{ZOO}$ & AGO-SET & OUT-NOV & 932 \\
\hline Eugenia aurata O.Berg & FAN & $\mathrm{ZOO}$ & OUT & - & 1120 \\
\hline Eugenia bimarginata DC. $(*)$ & FAN & $\mathrm{ZOO}$ & - & - & - \\
\hline Eugenia punicifolia (Kunth) DC. & FAN & $\mathrm{ZOO}$ & FEV & FEV & 1293 \\
\hline Eugenia livida O.Berg $(*)$ & FAN & $\mathrm{ZOO}$ & - & - & - \\
\hline Myrcia bella Cambess. & FAN & $\mathrm{ZOO}$ & - & NOV & 1161 \\
\hline Myrcia guianensis (Aubl.) DC. & FAN & $\mathrm{ZOO}$ & SET-NOV & NOV & 1004 \\
\hline Myrcia uberavensis O.Berg & FAN & $\mathrm{ZOO}$ & JUN-SET & - & 860 \\
\hline $\begin{array}{r}\text { Psidium cinereum Mart. ex DC. }(*) \\
\text { Nyctaginaceae }\end{array}$ & CAM & $\mathrm{ZOO}$ & - & - & - \\
\hline $\begin{array}{c}\text { Guapira noxia (Netto) Lundell }(*) \\
\text { Ochnaceae }\end{array}$ & FAN & AUT & - & - & - \\
\hline $\begin{array}{l}\text { Ouratea spectabilis (Mart.) Engl. } \\
\text { Polygalaceae }\end{array}$ & FAN & $\mathrm{ZOO}$ & AGO-SET & OUT & 929 \\
\hline $\begin{array}{l}\text { Bredemeyera floribunda Willd. } \\
\text { Portulacaceae }\end{array}$ & FAN & $\mathrm{ZOO}$ & FEV & - & 1289 \\
\hline $\begin{array}{l}\text { Portulaca mucronata } \text { Link } \\
\text { Rubiaceae }\end{array}$ & HEM & AUT & DEZ & - & 942 \\
\hline Alibertia sessilis (Vell.) K.Schum. & HEM & $\mathrm{ZOO}$ & AGO & - & 950 \\
\hline Borreria verticilata (L.) G.Mey. & HEM & AUT & JAN-MAR & - & 1321 \\
\hline Diodia teres Walter & TER & AUT & JAN & - & 808 \\
\hline Palicourea coriacea (Cham.) K.Schum. & HEM & $\mathrm{ZOO}$ & OUT-NOV & - & 1104 \\
\hline Palicourea rigida Kunth & CAM-FAN & $\mathrm{ZOO}$ & NOV & - & 1215 \\
\hline Rudgea virbunoides Benth. $(*)$ & FAN & $\mathrm{ZOO}$ & - & - & - \\
\hline $\begin{array}{c}\text { Tocoyena formosa (Cham. \& Schltdl.) K.Schum. } \\
\text { Sapindaceae }\end{array}$ & FAN & $\mathrm{ZOO}$ & OUT-NOV & FEV & 1115 \\
\hline Paullinia elegans Cambess. & LIA & ANE & SET & - & 1011 \\
\hline Serjania reticulata Cambess. & LIA & ANE & MAR-OUT & JUN & 840 \\
\hline Talisia angustifolia Radlk. & CAM & $\mathrm{ZOO}$ & OUT & NOV-DEZ & 1097 \\
\hline $\begin{array}{l}\text { Toulicia tomentosa } \text { Radlk. } \\
\text { Sapotaceae }\end{array}$ & CAM & ANE & MAI & JUN & 823 \\
\hline Pouteria ramiflora (Mart.) Radlk. & FAN & $\mathrm{ZOO}$ & MAI-AGO & - & 824 \\
\hline Pouteria subcaerulea Pierre ex Dubard & CAM & $\mathrm{ZOO}$ & SET-NOV & NOV & 1037 \\
\hline $\begin{array}{l}\text { Pouteria torta (Mart.) Radlk. } \\
\text { Solanaceae }\end{array}$ & FAN & $\mathrm{ZOO}$ & AGO-OUT & - & 971 \\
\hline $\begin{array}{l}\text { Solanum erianthum } \text { D.Don } \\
\text { Styracaceae }\end{array}$ & CAM & $\mathrm{ZOO}$ & OUT & - & 1153 \\
\hline $\begin{array}{r}\text { Styrax ferrugineus Nees \& Mart. } \\
\text { Verbenaceae }\end{array}$ & FAN & $\mathrm{ZOO}$ & MAR-MAI & - & 1349 \\
\hline Aegiphila lhotzkiana Cham. $(*)$ & CAM-FAN & $\mathrm{ZOO}$ & - & - & - \\
\hline $\begin{array}{l}\text { Lippia salviifolia Cham. } \\
\text { Vochysiaceae }\end{array}$ & CAM & AUT & FEV & - & 1294 \\
\hline Qualea dichotoma (Mart.) Warm. & FAN & ANE & - & MAR & 1337 \\
\hline Qualea grandiflora Mart. (*) & FAN & ANE & - & - & - \\
\hline Qualea multiflora Mart. & FAN & ANE & NOV-DEZ & - & 1226 \\
\hline Qualea parviflora Mart. (*) & FAN & ANE & - & - & - \\
\hline
\end{tabular}




\begin{tabular}{|c|c|c|c|c|c|}
\hline FAMÍLIA / ESPÉCIE & $\begin{array}{c}\text { Forma } \\
\text { de } \\
\text { vida }\end{array}$ & $\begin{array}{l}\text { Síndrome } \\
\text { de } \\
\text { dispersão }\end{array}$ & $\begin{array}{l}\text { Período } \\
\text { de } \\
\text { floração }\end{array}$ & $\begin{array}{l}\text { Período } \\
\text { de } \\
\text { frutificação }\end{array}$ & $\begin{array}{l}\text { Número do } \\
\text { coletor } \\
\text { SAPGodoy }\end{array}$ \\
\hline $\begin{array}{c}\text { Vochysia tucanorum Mart. } \\
\text { LILIOPSIDA } \\
\text { Arecaceae }\end{array}$ & FAN & ANE & NOV & - & 1227 \\
\hline Attalea geraensis Barb.Rodr. & GEO & $\mathrm{ZOO}$ & - & - & 1340 \\
\hline $\begin{array}{l}\text { Syagrus loefgrenii Glass } \\
\text { Bromeliaceae }\end{array}$ & CAM & $\mathrm{ZOO}$ & MAI & JAN & 829 \\
\hline Ananas ananassoides (Baker) L.B.Sm. & HEM & $\mathrm{ZOO}$ & NOV & MAR & 1164 \\
\hline Tillandsia pohliana $\mathrm{Mez}$ & EPI & AUT & - & FEV & 1296 \\
\hline $\begin{array}{l}\text { Tillandsia recurvata }(\mathrm{L} .) \mathrm{L} . \\
\text { Commmelinaceae }\end{array}$ & EPI & AUT & MAI & - & 833 \\
\hline $\begin{array}{l}\text { Commelina erecta } \mathrm{L} . \\
\text { Cyperaceae }\end{array}$ & TER & AUT & NOV-JUN & - & 895 \\
\hline Rhynchospora exaltata Kunth & HEM & AUT & SET & - & 1071 \\
\hline $\begin{array}{c}\text { Scleria comosa (Nees) Steud. } \\
\text { Liliaceae }\end{array}$ & HEM & AUT & - & MAR & 1358 \\
\hline $\begin{array}{l}\text { Alstroemeria pulchella } \text { L.f. } \\
\text { Orchidaceae }\end{array}$ & HEM & AUT & JAN-FEV & JAN & 1261 \\
\hline $\begin{array}{c}\text { Galeandra montana Barb.Rodr. } \\
\text { Poaceae }\end{array}$ & GEO & ANE & FEV-MAR & - & 1292 \\
\hline $\begin{array}{c}\text { Loudetiopsis chrysotrix (Nees) Conert } \\
\text { Smilacaceae }\end{array}$ & HEM & AUT & - & MAR & 1335 \\
\hline Smilax cissoides Mart. ex Griseb. & LIA & $\mathrm{ZOO}$ & SET-OUT & OUT-NOV & 1008 \\
\hline
\end{tabular}

\section{Agradecimentos}

À Fundação de Amparo à Pesquisa do Estado de São Paulo, pela bolsa de iniciação científica concedida à primeira autora (Processo $\mathrm{n}^{\circ}$ 97/07302-8).

\section{Referências bibliográficas}

Batalha, M.A. 1997. Análise da vegetação da ARIE - Cerrado Pé-de-Gigante (Santa Rita do Passa Quatro, SP). Dissertação de Mestrado. Universidade de São Paulo, São Paulo.

Batalha, M.A.; Aragaki, S. \& Mantovani, W. 1997a. Florística do cerrado em Emas, Pirassununga, SP. Boletim de Botânica da Universidade de São Paulo 16:49-64.

Batalha, M.A.; Aragaki, S. \& Mantovani, W. 1997 b. Variações fenológicas das espécies do cerrado em Emas (Pirassununga, SP). Acta Botanica Brasilica 11(1): 61-78.

Batalha, M.A. \& Mantovani, W. 2000. Reproductive phenological patterns of cerrado plant species at the Pe-de-Gigante reserve (Santa Rita do Passa Quatro, SP, Brazil): A comparison between the herbaceous and woody floras. Revista Brasileira de Biologia 60(1): 129-145.
Castro, A.A.J.F. 1987. Florística e fitossociologia de um cerrado marginal brasileiro, Parque Estadual de Vassununga, Santa Rita do Passa Quatro, SP. Dissertação de Mestrado. Universidade Estadual de Campinas, Campinas.

Castro, A.A.J.F.; Martins, F.R.; Tamashiro, J.Y. \& Shepherd, G.J. 1999. How rich is the flora of brazilian cerrados? Annals of the Missouri Botanical Gardens 86(1):192-224.

Cronquist, A. 1981. An integrated system of classification of flowering plants. Columbia University Press, New York.

Cronquist, A. 1988. The evolution and classification of flowering plants. $2^{\text {nd }}$ ed. The New York Botanical Garden, Bronx, New York.

Eiten, G. 1963. Habitat flora of fazenda Campininha, São Paulo, Brazil. In: Ferri, M.G. (coord). I Simpósio sobre o cerrado. EDUSP, São Paulo.

Felfili, J.M.; Silva Júnior, M.C.; Rezende, A.V.; Machado, J.W.B.; Walter, B.M.T.; Silva P.E.N. \& Hay, J.D. 1993. Análise comparativa da florística e fitossociologia da vegetação arbórea do cerrado sensu stricto na Chapada da Pratinha, DF- Brasil. Acta Botanica Brasilica 6(2): 27-46.

Goodland, R. 1969. Análise ecológica da vegetação de cerrado. In: Goodland, R. \& Ferri, M.G. Ecologia do cerrado. Itatiaia, Belo Horizonte e EDUSP, São Paulo. 
Mantovani, W. 1983. Composição e similaridade florística, fenologia e espectro biológico do cerrado na Reserva de Moji Guaçu, Estado de São Paulo. Dissertação de Mestrado, Universidade Estadual de Campinas, Campinas.

Mantovani, W. \& Martins, F.R. 1988.Variações fenológicas das espécies do cerrado da Reserva Biológica de Moji Guaçu, Estado de São Paulo. Revista Brasileira de Botânica 11(1/2): 101112.

Mantovani, W. \& Martins, F.R. 1993. Florística do cerrado da Reserva Biológica de Moji Guaçu, SP. Acta Botanica Brasilica 7(1): 33-60.

Martins, F.R. 1990. Atributos de comunidades vegetais. Quid, Teresina, 9(1/2): 12-17.

Mendonça, R.C. de; Felfili, J.M.; Walter, B.M.T.; Silva Júnior, M.C. da; Rezende, A.V.; Filgueiras, T.S. \& Nogueira, P.E. 1998. Flora Vascular do Cerrado. In: Sano, S.M. \& Almeida, S.P. 1998. Cerrado ambiente e flora. EMBRAPA-CPAC, Planaltina, GO. 289-556.

Oliveira, P.E. 1998. Fenologia e biologia reprodutiva das espécies de cerrado. In: Sano, S.M. \& Almeida, S.P. 1998. Cerrado: ambiente e flora. EMBRAPA-CPAC, Planaltina, GO 169-192.

Oliveira e Souza, M.H.A. 1977. Alguns aspectos ecológicos da vegetação na região perimetral da Represa do Lobo (Brotas-Itirapina, SP). Tese de Doutorado. Instituto de Biociências, Universidade de São Paulo, São Paulo.

Pagano, S.N.; César, O. \& Leitão Filho, H. de F. 1989. Composição florística do estrato arbustivoarbóreo da vegetação de cerrado da Área de Proteção Ambiental (APA) de Corumbataí - Estado de São Paulo. Revista Brasileira de Biologia 49(1): 37-48.

Pijl, L. van der. 1982. Principles of dispersal in higher plants. Springer-Verlag, Berlin.

Ratter, J.A.; Ribeiro, J.F. \& Bridgewater, S. 1997. The Brazilian cerrado vegetation and threats to its biodiversity. Annals of Botany 80(3): 223-230.

Ribeiro, J.F. \& Walter, B.M.T. 1998. Fitofisionomia do bioma Cerrado. In: Sano, S.M. \& Almeida, S.P. 1998. Cerrado ambiente e flora. EMBRAPACPAC, Planaltina, GO. 89-152.

Silberbauer-Gottsberger, I.; Morawetz, W. \& Gottsberger, G. 1977. Frost damage of cerrado plants in Botucatu, Brazil, as related to the geographical distribution of the species. Biotropica 9: 253-261.

Toledo Filho, D.V.; Leitão Filho, H. de F. \& Rodrigues, T.S. 1984. Composição florística de área de cerrado em Moji Mirim (SP). Boletim Técnico do Instituto Florestal 38: 165-175.

Warming, E. 1892. Lagoa Santa, contribuição para a geografia fitobiológica. In: Warming, E. \& Ferri, M.G. Lagoa Santa e a vegetação dos cerrados brasileiros. Itatiaia, Belo Horizonte e EDUSP, São Paulo. 